\title{
Riemann Problem for Shallow Water Equation with Vegetation
}

\author{
Stelian Ion, Dorin Marinescu, Stefan-Gicu Cruceanu
}

\begin{abstract}
We investigate the existence of the solution of the Riemann Problem for a simplified water flow model on a vegetated surface - system of shallow water type equations. It is known that the system with discontinuous topography is non-conservative even if the porosity is absent. A system with continuous topography and discontinuous porosity is also non-conservative. In order to define Riemann solution for such systems, it is necessary to introduce a family of paths that connects the states defining the Riemann Problem. We focus our attention towards choosing such a family based on physical arguments. We provide the structure of the solution for such Riemann Problems.
\end{abstract}

\section{Introduction}

We investigate the solution of the Riemann problem for the shallow water equation without friction but with porosity and topography

$$
\begin{aligned}
\frac{\partial}{\partial t}(\theta h)+\partial_{a}\left(\theta h v^{a}\right) & =0, \\
\frac{\partial}{\partial t}\left(\theta h v_{a}\right)+\partial_{b}\left(\theta h v_{a} v^{b}\right)+\theta h \partial_{a} w & =0, a=1,2,
\end{aligned}
$$

where $h(t, \boldsymbol{x})$ is the water height, $v^{a}(t, \boldsymbol{x})$ the components of the water velocity, $\theta(\boldsymbol{x})$ the porosity of the plant cover, and $z(\boldsymbol{x})$ the soil surface level. The

Key Words: hyperbolic nonconservativ law, measure solutions, path connectig, shock waves

2010 Mathematics Subject Classification: Primary 35L67; Secondary 35Q92, 76S05.

Received: December, 2016.

Revised: February, 2016.

Accepted: June, 2017. 
function $w=g(z+h)$ stands for the potential of the water level and $g$ is gravitational acceleration. The model (1) yields a conservative equation for the energy of water plant system

$$
\frac{\partial}{\partial t}(\theta h \varepsilon)+\frac{\partial}{\partial y^{a}}\left[\theta h v^{a}\left(\varepsilon+\frac{h}{2}\right)\right]=0 .
$$

The energy $\mathcal{E}$ is given by

$$
\mathcal{E}=\frac{1}{2}|v|^{2}+g\left(x^{3}+\frac{h}{2}\right) .
$$

The Riemann Problem is not only interesting from a mathematical point of view, but it has an important application in numerical methods. For the 1-D conservative hyperbolic systems

$$
\partial_{t} U+\partial_{x} F(U)=0, U \in \mathbb{R}^{n},
$$

the first existence results are due to Lax, [9]. Based on these results Glimm, [8] demonstrates the existence of the solution when the initial data has small total variation. The aforementioned existence results are based on the fundamental assumptions that there exits a domain $D \subset \mathbb{R}^{n}$ inside of which the Jacobian matrix $\partial_{U} F$ has $n$ distinct real eigenvalues $\lambda_{i}(U), i=\overline{1, n}$ and the right eigenvectors $r_{i}(U)$ satisfy one of the following conditions:

- the genuine nonlinear property $\nabla \lambda_{i}(U) \cdot r_{i}(U) \neq 0$

or

- the linear degenerated property $\nabla \lambda_{i}(U) \cdot r_{i}(U)=0$.

The Lax solution of the Riemann problem consists in at most $n+1$ constant states separated by shock or rarefaction waves. If we denote the solution by $W(t, x)$, then it satisfies the system (4) in the sense of distributions:

$$
\int_{0}^{\infty} \int_{-\infty}^{\infty}\left[W(t, x) \partial_{t} \phi+F(W) \partial_{x} \phi\right] \mathrm{d} t \mathrm{~d} x=0,
$$

for any smooth function $\phi(t, x)$ with compact support in $(0, \infty) \times(-\infty,+\infty)$.

For the case of a nonconservative hyperbolic systems

$$
\partial_{t} U+A(U) \partial_{x} U=0, U \in \mathbb{R}^{n},
$$

in order to define a Lax type solution of the Riemann problem, one needs to give a mathematical sense to the nonconservative product $A(U) \partial_{x}(U)$ when $U$ is a discontinuous function. One way to solve this problem was proposed in $[14,5]$ where two new concepts were introduced, namely path connecting and measure solutions. 
We now consider the following hyperbolic system of equations given in a more general form

$$
a_{j}^{i \alpha}(u) \partial_{\alpha} u^{j}=0,
$$

where $u: \mathbb{R}^{k} \rightarrow \mathbb{R}^{n}$. One assumes that the functions $a_{j}^{i \alpha}(\cdot)\left(a_{j}^{i \alpha}: D \subset \mathbb{R}^{n} \rightarrow\right.$ $\mathbb{R})$ are all smooth on a domain $D \subset \mathbb{R}^{n}$ and for any $\xi \in \mathbb{R}^{k} \neq 0$ the matrix $[\widetilde{a}(y) \xi]_{j}^{i}:=a_{j}^{i \alpha}(y) \xi_{\alpha}$ has $n$ real eigenvalues for any $y \in D$.

If $a_{j}^{i \alpha}(u)=\partial_{j} f^{i \alpha}(u)$ and if there exists a $C^{1}$-hypersurface $S$ along with a distributional solution $u$ that has jump discontinuity, then on $S$ the limits of $u$ must satisfy the Rankine-Hugoniot relations:

$$
\left(f^{i \alpha}\left(u^{+}\right)-f^{i \alpha}\left(u^{-}\right)\right) \nu_{\alpha}=0,
$$

where $\nu_{\alpha}(x)$ is the unitary normal to the hypersurface $S$. Let

$$
\Phi\left(s ; U_{1}, U_{2}\right):=\left(\phi^{1}\left(s ; U_{1}, U_{2}\right), \ldots, \phi^{n}\left(s ; U_{1}, U_{2}\right)\right)
$$

be a $C^{1}$ curve in $\mathbb{R}^{n}$ connecting the points $U_{1}$ and $U_{2}$, i.e.

$$
\Phi\left(0 ; U_{1}, U_{2}\right)=U_{1}, \quad \Phi\left(1 ; U_{1}, U_{2}\right)=U_{2} .
$$

Using the path $\Phi(\cdot)$, one can write

$$
\begin{aligned}
\left(f^{i \alpha}\left(u^{+}\right)-f^{i \alpha}\left(u^{-}\right)\right) \nu_{\alpha} & =\left(f^{i \alpha}\left(\Phi\left(1 ; u^{-}, u^{+}\right)\right)-f^{i \alpha}\left(\Phi\left(0 ; u^{-}, u^{+}\right)\right)\right) \nu_{\alpha} \\
& =\int_{0}^{1} \frac{d}{\mathrm{~d} s} f^{i \alpha}\left(\Phi\left(s ; u^{-}, u^{+}\right)\right) \nu_{\alpha} \mathrm{d} s \\
& =\int_{0}^{1} a_{j}^{i \alpha}\left(\Phi\left(s ; u^{-}, u^{+}\right)\right) \frac{d}{\mathrm{~d} s} \phi^{j}\left(s ; u^{-}, u^{+}\right) \nu_{\alpha} \mathrm{d} s \\
& =0 .
\end{aligned}
$$

A piecewise smooth function $u(x)$ can be defined as a solution of the nonconservative system (7) if one gives a sense to the product $a \partial u$.

Let $\Phi\left(s ; U_{1}, U_{2}\right)$ be a connecting path in sense of [5] and $u \in S B V\left(\mathbb{R}^{k}, \mathbb{R}^{n}\right)$, where $S B V\left(\mathbb{R}^{k}, \mathbb{R}^{n}\right)$ represents the space of special bounded variation functions [1]. The derivative $\partial u$ in a distributional sense of $u \in S B V\left(\mathbb{R}^{k}, \mathbb{R}^{n}\right)$ is a Radon measure on $\mathbb{R}^{k}$ with finite total variation. There exists a set $J_{u}$ of jump points of $u$ (see Figure (1)) such that

$$
\partial u=\nabla u \mathcal{L}^{k}+\left(u^{+}-u^{-}\right) \nu \mathcal{H}^{k-1}\left\llcorner J_{u},\right.
$$




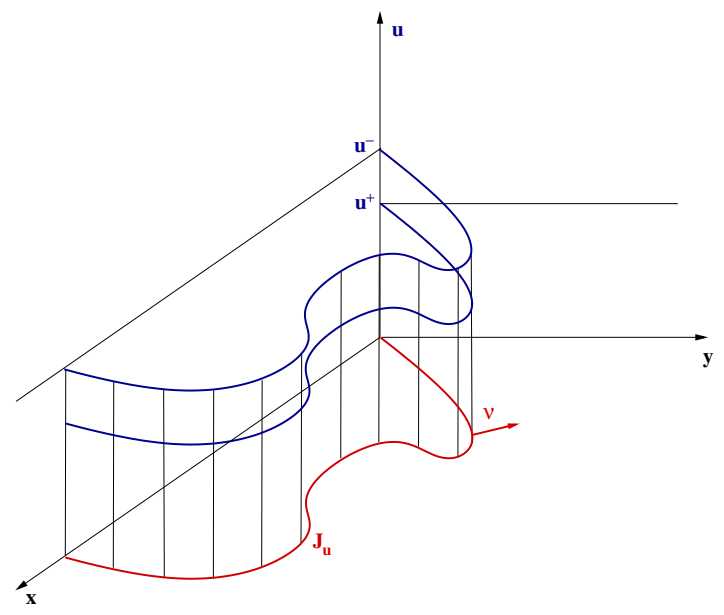

Figure 1: Jump of $u$.

where $\mathcal{L}^{k}$ designates the Lebesgue measure on $\mathbb{R}^{k}$. The measure $\mathcal{H}^{k-1}\left\llcorner J_{u}\right.$ represents the $(k-1)$-Hausdorff measure on $\mathbb{R}^{k}$ restricted to the set $J_{u}$. Componentwise written, (10) reads as

$$
(\partial u)_{\alpha}^{i}=\left(\partial_{\alpha} u^{i}\right) \cdot \mathcal{L}^{k}+\left(\left(u^{i+}-u^{i-}\right) \nu_{\alpha}\right) \cdot \mathcal{H}^{k-1}\left\llcorner J_{u} .\right.
$$

Using the notion of path connecting $\Phi\left(s ; U_{1}, U_{2}\right)$, one can now define the nonconservative product $a(u) \partial u$ as the measure

$$
a(u) \partial u=a(u) \nabla u \mathcal{L}^{k}+\left[\int_{0}^{1} a\left(\Phi\left(s ; u^{+}, u^{-}\right)\right) \frac{d}{\mathrm{~d} s} \phi\left(s ; u^{+}, u^{-}\right) \mathrm{d} s\right] \nu \mathcal{H}^{k-1}\left\llcorner J_{u} .\right.
$$

In (12), the measure $a(u) \nabla u \mathcal{L}^{k}$ is the absolute continuous component (w.r.t. the Lebesgue measure $\mathcal{L}^{k}$ ) of $a \partial u$. The remaining terms in (12) correspond to the singular part of $a \partial u$ (w.r.t. the Hausdorff measure $\mathcal{H}^{k-1}$ ) that is concentrated on the $(k-1)$-dimensional surface $J_{u}$. (In definition (12) we assume that $a \partial u$ has no singular continuous part.)

Componentwise, (12) has the following form

$$
\begin{aligned}
(a \partial u)^{i} & =a_{j}^{i \alpha}(u)\left(\partial_{\alpha} u^{j}\right) \cdot \mathcal{L}^{k}+ \\
& +\left[\int_{0}^{1} a_{j}^{i \alpha}\left(\Phi\left(s ; u^{+}, u^{-}\right)\right) \frac{d}{\mathrm{~d} s} \phi^{j}\left(s ; u^{+}, u^{-}\right) \mathrm{d} s\right] \nu_{\alpha} \cdot \mathcal{H}^{k-1}\left\llcorner J_{u} .\right.
\end{aligned}
$$


Definition 1.1. We say that a function $u \in S B V\left(\mathbb{R}^{k}, \mathbb{R}^{n}\right)$ is a solution of the nonconservative system (7) if the measure a(u)du defined by (12) satisfies

$$
a(u) \partial u(B)=0 .
$$

for any Borel set $B$.

For regular points (14) gives

$$
a(u) \nabla u=0,
$$

while for the points belonging to $J_{u}$ one has the generalized Rankine-Hugoniot relations

$$
\left[\int_{0}^{1} a\left(\Phi\left(s ; u^{+}, u^{-}\right) \frac{d}{\mathrm{~d} s} \phi\left(s ; u^{+}, u^{-}\right) \mathrm{d} s\right] \nu=0 .\right.
$$

Remark 1.1. The definition 1.1 of a measure solution $u$ assumes the existence of the connecting path $\Phi$ between the jump values $u^{-}$and $u^{+}$of $u$ on the discontinuity set $J_{u}$. As a consequence, the function $\Phi$ is part of the model (7). The choice of a most suited path $\Phi$ is a modeling problem. For the shallow water equations, we can choose a class of connecting path functions $\Phi$ having the property that the jump relations are independent of the path $\Phi$ and we argue the opportunity of this selection by invoking physical arguments.

For shallow water equations (1), the functions $\theta(x)$ and $z(x)$ are given data and they are not part of the unknowns of the problem. But when the terrain data are discontinuous, defining the solution becomes a problem. In order to manage the discontinuity of this solution, one also considers the terrain data as unknowns of the problem [10,3] and extend the system (1) by adding two new equations

$$
\begin{aligned}
& \partial_{t} \theta=0, \\
& \partial_{t} z=0 .
\end{aligned}
$$

We now consider as unknown the vector $\boldsymbol{u}=\left(h, v^{1}, v^{2}, \theta, z\right)^{\mathrm{T}}$. Denoting the time variable $t$ by $x^{0}$, the extended system of equations $(1)+(17)$ can be compactly written as

$$
\partial_{\alpha} f^{I \alpha}(\boldsymbol{u})+d^{I \alpha}(\boldsymbol{u}) \partial_{\alpha} w=0
$$

where $I \in\{1, \ldots, 5\}$ and $\alpha \in\{0,1,2\}$ (with $\partial_{0}:=\partial_{t}, \partial_{1}:=\partial_{x^{1}}, \partial_{2}:=\partial_{x^{2}}$ ). In (18), $f^{I \alpha}$ and $d^{I \alpha}$ are the components of the matrices

$$
f(\boldsymbol{u})=\left[\begin{array}{ccc}
\theta h & \theta h v^{1} & \theta h v^{2} \\
\theta h v^{1} & \theta h v^{1} v^{1} & \theta h v^{2} v^{1} \\
\theta h v^{2} & \theta h v^{1} v^{2} & \theta h v^{2} v^{2} \\
\theta & 0 & 0 \\
z & 0 & 0
\end{array}\right] \text { and } d(\boldsymbol{u})=\left[\begin{array}{ccc}
0 & 0 & 0 \\
0 & \theta h & 0 \\
0 & 0 & \theta h \\
0 & 0 & 0 \\
0 & 0 & 0
\end{array}\right]
$$


respectively.

\subsection{Nonsteady jump surface}

Consider a jump surface $s\left(t, x^{1}, x^{2}\right)=0$ of a measure solution $\boldsymbol{u}$. The normal to the surface $s$ has the direction defined by $\left(\partial_{t} s, \partial_{1} s, \partial_{2} s\right)$.

The jump of a solution of (18) must satisfy the relations

$$
\left(f^{I \alpha}\left(\boldsymbol{u}^{+}\right)-f^{I \alpha}\left(\boldsymbol{u}^{-}\right)\right) \nu_{\alpha}+g\left[\int_{0}^{1} d^{I \alpha}(\theta(s), h(s)) \frac{d(z(s)+h(s))}{\mathrm{d} s} \mathrm{~d} s\right] \nu_{\alpha}=0 .
$$

The last two equations of (20) lead to

$$
\left(z^{+}-z^{-}\right) \partial_{t} s=0, \quad\left(\theta^{+}-\theta^{-}\right) \partial_{t} s=0 .
$$

From $(21)$ one can observe that a nonsteady jump surface (i.e. $\partial_{t} s \neq 0$ ) can only propagate in a smooth region of terrain data (i.e. $z^{+}-z^{-}=0$ and $\left.\theta^{+}-\theta^{-}=0\right)$. For such jump surface, $(h, \boldsymbol{v})$ satisfy

$$
\begin{aligned}
-\sigma[|h|]+\left[\left|h v^{\perp}\right|\right] & =0, \\
-\sigma\left[\left|h v^{a}\right|\right]+\left[\left|h v^{a} v^{\perp}\right|\right]+g \frac{\left[\left|h^{2}\right|\right]}{2} n^{a} & =0, \quad\left(n^{a}:=\partial_{a} s / \| \nabla s||\right),
\end{aligned}
$$

where $\sigma=-\partial_{t} s /\|\nabla s\|$ is the displacement velocity of the jump surface and $v^{\perp}=v^{a} \partial_{a} s /\|\nabla s\|$ is normal component of the fluid velocity to the jump surface. For a function $\psi,[|\psi|]$ stands for the jump $\psi^{+}-\psi^{-}$.

Projecting the jump relations (22) onto the normal and tangential direction of $s$, one obtains

$$
\begin{aligned}
-\sigma[|h|]+\left[\left|h v^{\perp}\right|\right] & =0, \\
-\sigma\left[\left|h v^{\perp}\right|\right]+\left[\left|h\left(v^{\perp}\right)^{2}+g h^{2} / 2\right|\right] & =0, \\
h^{-}\left(v^{\perp-}-\sigma\right)\left[\left|v^{\|}\right|\right] & =0 .
\end{aligned}
$$

The last equation shows that if $v^{\perp} \neq \sigma$, then the tangential component of the velocity of the fluid is a continuous function.

\section{$1.2 \quad$ Steady jump surface}

Using (21), one can easily observe that if $z^{+}-z^{-} \neq 0$ or $\theta^{+}-\theta^{-} \neq 0$, then $\partial_{t} s=0$, which means that a jump in the terrain surface, plant cover density, or both generates a steady jump surface for a measure solution. 
Let $s\left(x^{1}, x^{2}\right)=0$ be a jump curve of the terrain data. The jump relations now become

$$
\begin{aligned}
{\left[\left|\theta h v^{\perp}\right|\right] } & =0, \\
{\left[\left|\theta h v^{a} v^{\perp}\right|\right]+g n^{a} \int_{0}^{1} \theta(s) h(s) \frac{d(z(s)+h(s))}{\mathrm{d} s} \mathrm{~d} s } & =0,
\end{aligned}
$$

and then

$$
\begin{aligned}
{\left[\left|\theta h v^{\perp}\right|\right] } & =0, \\
{\left[\left|\theta h\left(v^{\perp}\right)^{2}\right|\right]+g \int_{0}^{1} \theta(s) h(s) \frac{d(z(s)+h(s))}{\mathrm{d} s} \mathrm{~d} s } & =0, \\
\theta h\left(v^{\perp}\right)^{-}\left[\left|v^{\|}\right|\right] & =0 .
\end{aligned}
$$

Relations (25) result from (24) in a similar manner as (23) are deduced from (22). As in the non-steady case, the jump of tangential component of the velocity is zero when $v^{\perp}=0,[5,6,14]$.

\section{Planar Oblique Waves}

We are now looking for a particular class of solutions of the shallow water equations: the oblique waves. This means that the solution only depends on $t$ and $\xi=\boldsymbol{x} \cdot \boldsymbol{n}$, where $\boldsymbol{n}$ is a given unitary vector. The system of equations (1) written for oblique waves solutions has the following form

$$
\begin{aligned}
\partial_{t}(\theta h)+\partial_{\xi}(\theta h v) & =0, \\
\partial_{t}(\theta h v)+\partial_{\xi}\left(\theta h v^{2}\right)+\theta h \partial_{\xi} w & =0, \\
\partial_{t}(\theta h v)+\partial_{\xi}(\theta h v \underline{v}) & =0,
\end{aligned}
$$

where $v=v^{1}$ and $\underline{v}=v^{2}$ are the orthogonal and tangential components of the velocity to the plane $\boldsymbol{x} \cdot \boldsymbol{n}$, respectively.

Observe that $h$ and $v$ do not depend on the tangential component of velocity $\underline{v}$; also this $\underline{v}$ can be very easily calculated if one knows $h$ and $v$. We introduce the operator

$$
\mathcal{H}(h, v)=\partial_{t}(\theta h v)+\partial_{\xi}\left(\theta h v^{2}\right)+\theta h \partial_{\xi} w
$$

as a measure of the linear momentum balance. By standard calculations, one can also write

$$
\mathcal{H}(h, v)=\theta h\left(\partial_{t} v+\partial_{\xi}\left(v^{2} / 2+w\right)\right)+v\left(\partial_{t}(\theta h)+\partial_{\xi}(\theta h v)\right) .
$$


If $h$ and $v$ are smooth functions and if they verify the following conservative system of equations

$$
\begin{aligned}
\partial_{t}(\theta h)+\partial_{\xi}(\theta h v) & =0, \\
\partial_{t} v+\partial_{\xi}\left(v^{2} / 2+w\right) & =0,
\end{aligned}
$$

then the balance of linear momentum is also satisfied. In contrast, if $h$ and $v$ are shock wave solutions of the conservative equations, then the the balance of linear momentum is not satisfied. This means that the two systems (26) and (27) are not equivalent, in the sense that they furnish different solutions in the class of $S B V(\mathbb{R}, \mathbb{R})$ functions. The shock wave solutions obtained by solving (27) are not physically suitable.

Proposition 2.1. Let $\Phi\left(s ; U_{1}, U_{2}\right)$ be a path connecting function in $\mathbb{R}^{2}$ and $(h, v, \theta, z)(\xi)$ a steady shock solution of $(27)$ i.e.

$$
\begin{aligned}
{[|\theta h v|] } & =0, \\
{\left[\left|v^{2} / 2+w\right|\right] } & =0 .
\end{aligned}
$$

It follows that

$$
\mathcal{H}(h, v) \neq 0 .
$$

Proof. On the shock wave curve $(t, x)=(t, 0)$ one has

$$
\mathcal{H}(h, v)=\int_{0}^{1}\left(\theta(s) h(s) \frac{\mathrm{d}\left(v^{2} / 2(s)+w(s)\right)}{\mathrm{d} s}+v(s) \frac{\mathrm{d} \theta(s) h(s) v(s)}{\mathrm{d} s}\right) \mathrm{d} s .
$$

Therefore, $\mathcal{H}(h, v)=0$ only for very special paths $\Phi$.

In contrast with [7], we consider that at a jump point $\xi_{0}$ one has

$$
\begin{array}{r}
{[|\theta h v|]=0,} \\
{\left[\left|\theta h v^{2}\right|\right]+g \int_{0}^{1} \theta(s) h(s) \frac{\mathrm{d}(z(s)+h(s))}{\mathrm{d} s} \mathrm{~d} s=0 .}
\end{array}
$$

In what follows, we will consider two different cases:

1. soil surface discontinuity and continuous porosity,

2. jumps in soil surface and porosity. 


\subsection{Case 1. Steady soil surface jump and continuous porosity}

Let $\xi_{0}$ be a jump point. Denote the left and right limit values of a function by $L$ and $R$ superscripts, respectively. The generalized Rankine-Hugoniot jump relations (30) take the form

$$
\begin{aligned}
& {[|h v|]=0,} \\
& {\left[\left|h v^{2}+h^{2} / 2\right|\right]+g \int_{0}^{1} h\left(s ; h^{L}, h^{R}\right) \frac{\mathrm{d} z\left(s ; z^{L}, z^{R}\right)}{\mathrm{d} s} \mathrm{~d} s=0 .}
\end{aligned}
$$

Our purpose is to choose physical relevant path $\left(h\left(s ; h^{L}, h^{R}\right), z\left(s ; z^{L}, z^{R}\right)\right)$ such that the integral in (31) to be analytically evaluated. The easiest choice would be the segment path. Though, we can use a larger class of paths of the following form

$$
\begin{aligned}
& h\left(s ; h^{L}, h^{R}\right)=h^{L}+\phi(s)\left(h^{R}-h^{L}\right), \\
& z\left(s ; h^{L}, h^{R}\right)=z^{L}+\phi(s)\left(z^{R}-z^{L}\right),
\end{aligned}
$$

where $\phi(s)$ can be any smooth function that satisfies $\phi(0)=0$ and $\phi(1)=1$. For arbitrary such $\phi$ in (32) one has

$$
\int_{0}^{1} h\left(s ; h^{L}, h^{R}\right) \frac{\mathrm{d} z\left(s ; z^{L}, z^{R}\right)}{\mathrm{d} s} \mathrm{~d} s=\frac{h^{L}+h^{R}}{2}[|z|],
$$

and therefore the above integral is independent of $\phi$. The choice of the path

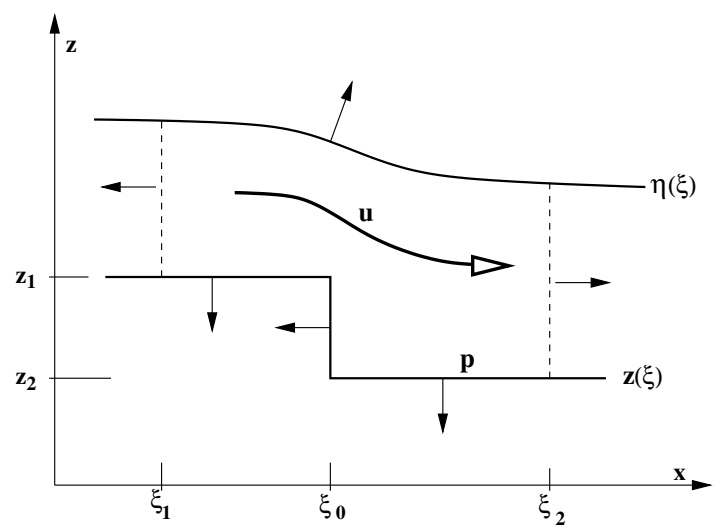

Figure 2: Balance of linear momentum.

(32) can be justified by certain physical considerations. 
Consider a fluid volume $\Omega=\left\{(\xi, z) \mid \xi_{1} \leq \xi \leq \xi_{2}, z(\xi)<z<\eta(\xi)\right\}$, see figure 2, where $z(\xi)$ is the soil surface altitude and $\eta(\xi)$ is the water surface level. For the case of steady motion and in the absence of any frictional forces, the integral form of the linear momentum relatively to $\Omega$ is given by

$$
\int_{\partial \Omega} \widetilde{v} \widetilde{\boldsymbol{v}} \cdot \boldsymbol{\nu} \mathrm{d} \sigma+\int_{\partial \Omega} p \nu_{x} \mathrm{~d} \sigma=0
$$

where $\widetilde{\boldsymbol{v}}(\xi, z)$ is the fluid velocity $(\widetilde{\boldsymbol{v}}=(\widetilde{v}, \underline{\widetilde{v}})), p$ is the pressure of the fluid, $\boldsymbol{\nu}$ is the unitary normal to the boundary $\partial \Omega$ pointing outside $\Omega$, and $\mathrm{d} \sigma$ is the surface element on $\partial \Omega$. In the approximation of the shallow water equations, the pressure $p$ depends linearly on the water depth.

$$
p(\xi, z)=p_{\infty}+g(\eta(\xi)-z) .
$$

The "micro-local fluid velocity" $\widetilde{v}$ is averaged along water depth. We use the notation

$$
h v^{2}(\xi):=\int_{0}^{h(\xi)} \widetilde{v}^{2}(\xi, z) \mathrm{d} z,
$$

where $h(\xi)=\eta(\xi)-z(\xi)$, with $\eta(\xi)$ representing the level of the free water surface (see Fig. 2).

Since $\nu_{x}=0$ on the boundary parts $\xi_{1}, \xi_{0}$ and $\xi_{0}, \xi_{2}$, using (34) and (35), the integral relation (33) becomes

$$
\begin{array}{r}
h v^{2}\left(\xi_{2}\right)-h v^{2}\left(\xi_{1}\right)+g \int_{z\left(\xi_{2}\right)}^{\eta\left(\xi_{2}\right)}\left(\eta\left(\xi_{2}\right)-z\right) \mathrm{d} z-g \int_{z\left(\xi_{1}\right)}^{\eta\left(\xi_{2}\right)}\left(\eta\left(\xi_{1}\right)-z\right) \mathrm{d} z- \\
-g \int_{z_{2}}^{z_{1}}\left(\eta\left(\xi_{0}\right)-z\right) \mathrm{d} z=0 .
\end{array}
$$

\subsection{Case 2. Steady soil surface jump and porosity jump}

The presence of a simultaneous discontinuity in the cover plant and soil surface makes the Riemann Problem more complicated when compared to the previous case. The first question is how to choose a path that connects the states separated by discontinuity. We look again for an answer with physical significance for our choice. For this purpose, we remember that the mathematical model of water flow on a vegetated hill uses averaged quantities $h, \boldsymbol{u}$. The average is made along water depth, but also with respect to the plan soil surface domain. 
The water depth $h$ is defined as

$$
h(x, t)=\frac{1}{\omega_{f}} \int_{D_{f}} \widetilde{h}(y, t) \mathrm{d} y,
$$

where $D$ is the averaging window, $D_{f}$ is the interface fluid-soil inside $D, \widetilde{h}(y, t)$ is micro-local water depth, and $\omega_{f}$ is the area of $D_{f}$. If one imagines a sharp transition between two domains characterized by $\omega_{f}^{L}, \widetilde{h}^{L}$ and $\omega_{f}^{R}, \widetilde{h}^{R}$ then the averaged $h$ is given by

$$
h(x(s), t)=\frac{\widetilde{h}^{L} a(s)+\widetilde{h}^{R} b(s)}{a(s)+b(s)} .
$$

Here $b(s)=\omega_{f}^{R} \alpha(s), a(s)=\omega_{f}^{L}(1-\alpha(s))$, where $\alpha(s)$ represents the proportion of $\Omega^{R}$ domain (characterized by $\left.\omega_{f}^{R}\right)$ in $D$, (with $\alpha(1)=1$ and $\left.\alpha(0)=0\right)$. One observes that the area of $D_{f}$ is

$$
\omega_{f}(s)=\omega_{f}^{L}(1-\alpha(s))+\omega_{f}^{R} \alpha(s) .
$$

Simple algebraic calculations lead to

$$
h(s)=h^{L}+[|h|] \phi(s)
$$

and

$$
\theta(s)=\theta^{L}+[|\theta|] \alpha(s),
$$

where the functions $\alpha(s)$ and $\phi(s)$ are related through

$$
\alpha(s)=\phi(s) \frac{\theta^{L}}{\theta^{R}-\phi(s)[|\theta|]} .
$$

We define the path

$$
\begin{aligned}
& h\left(s ; h^{L}, h^{R}\right)=h^{L}+\phi(s)\left(h^{R}-h^{L}\right), \\
& z\left(s ; z^{L}, z^{R}\right)=z^{L}+\phi(s)\left(z^{R}-z^{L}\right), \\
& \theta\left(s ; \theta^{L}, \theta^{R}\right)=\theta^{L}+\alpha(s)\left(\theta^{R}-\theta^{L}\right), \\
& \alpha(s)=\phi(s) \frac{\theta^{L}}{\theta^{R}-\phi(s)\left(\theta^{R}-\theta^{L}\right)},
\end{aligned}
$$

where $\phi$ is an arbitrary smooth and monotone function that verifies $\phi(0)=0$ and $\phi(1)=1$. Applying the jump relations (25) to the path (37) one has

$$
u^{R}=\frac{u^{L}}{\theta h}
$$


and

$$
\operatorname{Fr}_{L}^{2}\left(\frac{1}{\theta h}-1\right)-[a(\theta)+b(\theta)(h-1)] \cdot(\lfloor|z|\rfloor+h-1)=0,
$$

where we used the notations

$$
\begin{aligned}
& h:=\frac{h^{R}}{h^{L}}, \quad \theta:=\frac{\theta^{R}}{\theta^{L}}, \quad\lfloor|z|\rfloor:=\frac{z^{R}-z^{L}}{h^{L}}, \\
& a(\theta):=\frac{\theta \ln \theta}{1-\theta}, \quad b(\theta):=\frac{\theta}{\theta-1}(1+a(\theta)), \\
& \operatorname{Fr}_{L}^{2}:=\frac{\left(u^{L}\right)^{2}}{g^{L}} .
\end{aligned}
$$

The problem now is to solve (39) for the unknown $h$ and for given $\theta, \operatorname{Fr}_{L}^{2}$ and $\lfloor|z|\rfloor$. The equation (39) is equivalent to

$$
-b(\theta) h^{3}-(a(\theta)-b(\theta)(1-\lfloor|z|\rfloor)) h^{2}+\left((1-\lfloor|z|\rfloor) a(\theta)-\operatorname{Fr}_{L}^{2}\right) h+\frac{\operatorname{Fr}_{L}^{2}}{\theta}=0,
$$

a cubic equation in $h$, where

$$
a(\theta)=\frac{-\theta(\theta-1-\ln \theta)}{(\theta-1)^{2}}, \quad b(\theta)=\frac{\theta(\theta-1-\theta \ln \theta)}{(\theta-1)^{2}} .
$$

Lemma 2.1. The functions $a(\theta), b(\theta):(0, \infty) \backslash\{1\} \rightarrow \mathbb{R}$ satisfy

$$
\begin{array}{cl}
\lim _{\theta \rightarrow 1} a(\theta)=-\frac{1}{2}, & \lim _{\theta \rightarrow 1} b(\theta)=-\frac{1}{2}, \\
a(\theta)<0, & b(\theta)<0 .
\end{array}
$$

Proof. This result can be easily proved using the inequalities

$$
x-1-x \ln x \leq 0, \quad x-1-\ln x \geq 0, \quad \forall x>0
$$

and the obvious limit

$$
\lim _{x \rightarrow 1} \frac{\ln x}{x-1}=1 .
$$

We now proceed to analyze the solutions of the equation (41). Introducing the function $\psi$ depending on the variable $h$ and parameters $\theta,\lfloor|z|\rfloor, \operatorname{Fr}_{L}$

$$
\begin{aligned}
\psi\left(h ; \theta,\lfloor|z|\rfloor, \operatorname{Fr}_{L}\right):= & -b(\theta) h^{3}-[a(\theta)-b(\theta)(1-\lfloor|z|\rfloor)] h^{2}+ \\
& +\left[(1-\lfloor|z|\rfloor) a(\theta)-\operatorname{Fr}_{L}^{2}\right] h+\frac{\operatorname{Fr}_{L}^{2}}{\theta},
\end{aligned}
$$

the equation (41) can be compactly written as

$$
\psi\left(h ; \theta,\lfloor|z|\rfloor, \operatorname{Fr}_{L}\right)=0 .
$$


Lemma 2.2. If $1-\lfloor|z|\rfloor>0$, then the equation

$$
\partial_{h} \psi\left(h ; \theta,\lfloor|z|\rfloor, \operatorname{Fr}_{L}\right)=0
$$

has two real solutions $\widetilde{h}_{1}<0<\widetilde{h}_{2}$.

Proof. The equation $\partial_{h} \psi(\cdot)=0$ can be written as

$$
-3 b(\theta) h^{2}-2[a(\theta)-b(\theta)(1-\lfloor|z|\rfloor)] h+a(\theta)(1-\lfloor|z|\rfloor)-\operatorname{Fr}_{L}^{2}=0
$$

and has two real solutions if

$$
\triangle:=[a-b(1-\lfloor|z|\rfloor)]^{2}+3 b\left[(1-\lfloor|z|\rfloor) a-\operatorname{Fr}_{L}^{2}\right]>0 .
$$

One can observe that

$$
\triangle=(a / 2+b(1-\lfloor|z|\rfloor))^{2}+3 a^{2} / 4-3 b \mathrm{Fr}_{L}^{2},
$$

which obviously is a positive quantity, since $b(\theta) \leq 0$. The solutions $\widetilde{h}_{1}, \widetilde{h}_{2}$ verify

$$
\widetilde{h}_{1} \cdot \widetilde{h}_{2}=\frac{(1-\lfloor|z|\rfloor) a-\mathrm{Fr}_{L}^{2}}{-3 b} \leq 0 .
$$

Then, the positive solution is

$$
\widetilde{h}_{2}=\frac{a(\theta)-b(\theta)(1-\lfloor|z|\rfloor)+\sqrt{\triangle}}{-3 b(\theta)} .
$$

In the following proposition we investigate the existence of the solutions for equation (46). We only consider the case of continuous fluid domain. More precisely,

Case 1. either $z^{R}-z^{L}>0$ and then one must satisfy $h^{L}>z^{R}-z^{L}$, i.e.

$$
1-\lfloor|z|\rfloor>0 ;
$$

Case 2. or $z^{R}-z^{L}<0$ and then one must satisfy $h^{R}>z^{L}-z^{R}$, i.e.

$$
h+\lfloor|z|\rfloor>0 .
$$

Remark that the inequality (50) is trivially satisfied in Case 1, and the inequality (49) is trivially satisfied in Case 2. Also, (49) is a condition on parameters, while (50) is a condition on solutions of (47). 
Proposition 2.2. Assume that $1-\lfloor|z|\rfloor>0$.

(a) Consider some given positive values for the parameters $\theta$ and $\mathrm{Fr}_{L}$. Let $\widetilde{h}_{2}$ be as in (48), the positive solution of (47).

If

$$
\left.\psi\left(\widetilde{h}_{2} ; \theta,\lfloor|z|\rfloor, \operatorname{Fr}_{L}\right)\right|_{\lfloor|z|\rfloor=0}<0,
$$

then there exists $\delta \in[0,1)$ such that equation (46) has two positive solutions $h_{1}<\widetilde{h}_{2}<h_{2}$, for all $\lfloor|z|\rfloor<\delta$.

(b) Equation (46) has two positive solutions for all $\theta$ and $\lfloor|z|\rfloor$ satisfying the inequality $1 / \theta<1-\lfloor|z|\rfloor$.

(c) If $\operatorname{Fr}_{L} \neq 1$, then there exist neighborhoods $\mathcal{V}_{1}(1)$ of 1 and $\mathcal{V}_{2}(0)$ of 0 such that equation (46) has two positive solutions for all $\theta \in \mathcal{V}_{1}(1)$ and $\lfloor|z|\rfloor \in \mathcal{V}_{2}(0)$. Moreover,

$$
\begin{array}{lll}
\lim _{\theta \rightarrow 1,\lfloor|z|\rfloor \rightarrow 0} h_{2}=1, & \text { if } & \operatorname{Fr}_{L}<1, \\
\lim _{\theta \rightarrow 1,\lfloor|z|\rfloor \rightarrow 0} h_{1}=1, & \text { if } & \operatorname{Fr}_{L}>1 .
\end{array}
$$

Proof. (a) Using the properties (44) of the coefficients $a(\theta), b(\theta)$ and Lemma 2.2 , the equation has two positive solutions only in the case

$$
\psi\left(\widetilde{h}_{2} ; \theta,\lfloor|z|\rfloor, \operatorname{Fr}_{L}\right)<0 .
$$

Since

$$
\begin{aligned}
& \frac{\mathrm{d}}{\mathrm{d}\lfloor|z|\rfloor} \psi\left(\widetilde{h}_{2} ; \theta,\lfloor|z|\rfloor, \operatorname{Fr}_{L}\right)= \\
& =\partial_{h} \psi\left(\widetilde{h}_{2} ; \theta,\lfloor|z|\rfloor, \operatorname{Fr}_{L}\right) \cdot \partial_{\lfloor|z|\rfloor} \widetilde{h}_{2}+\partial_{\lfloor|z|\rfloor} \psi\left(\widetilde{h}_{2} ; \theta,\lfloor|z|\rfloor, \operatorname{Fr}_{L}\right)=0 \cdot \partial_{\lfloor|z|\rfloor} \widetilde{h}_{2}- \\
& b(\theta) \widetilde{h}_{2}^{2}-a(\theta) \widetilde{h}_{2}=-b(\theta) \widetilde{h}_{2}^{2}-a(\theta) \widetilde{h}_{2}
\end{aligned}
$$

it follows that

$$
\frac{\mathrm{d}}{\mathrm{d}\lfloor|z|\rfloor} \psi\left(\widetilde{h}_{2} ; \theta,\lfloor|z|\rfloor, \operatorname{Fr}_{L}\right)=-b(\theta) \widetilde{h}_{2}^{2}-a(\theta) \widetilde{h}_{2}>0
$$

and consequently

$$
\begin{aligned}
& \left.\psi\left(\widetilde{h}_{2} ; \theta,\lfloor|z|\rfloor, \operatorname{Fr}_{L}\right)\right|_{\lfloor|z|\rfloor=1} \geq\left.\psi\left(\widetilde{h}_{2} ; \theta,\lfloor|z|\rfloor, \operatorname{Fr}_{L}\right)\right|_{\lfloor|z|\rfloor \in(0,1)} \geq \\
& \geq\left.\psi\left(\widetilde{h}_{2} ; \theta,\lfloor|z|\rfloor, \operatorname{Fr}_{L}\right)\right|_{\lfloor|z|\rfloor=0} .
\end{aligned}
$$

To prove the limits from (52), one extends by continuity with respect to $\theta$ the functions $a(\theta)$ and $b(\theta)$ and define

$$
\psi\left(h ; 1,0, \operatorname{Fr}_{L}\right)=1 / 2(h-1)\left(h^{2}+h-2 \operatorname{Fr}_{L}^{2}\right) .
$$


For any $\operatorname{Fr}_{L} \neq 1$, the function $\psi\left(h ; 1,0, \mathrm{Fr}_{L}\right)$ has two positive and distinct roots:

$$
\bar{h}_{1}=1 \text { and } \bar{h}_{2}=\frac{-1+\sqrt{1+8 \mathrm{Fr}_{L}^{2}}}{2} .
$$

One now can easily obtain (52) using continuity arguments.

Note that if $\operatorname{Fr}_{L}=1$, then we may not have positive solutions.

Since the solution of jump equation is not unique, we must select one that is physically relevant. In literature (e.g. [13]), two criteria are usually used and applied across of steady shock where,

1. the energy of fluid must not increase;

2. the soil altitude $z$ and the water depth $h$ must have the same monotonicity.

Here, when selecting the solution, if both porosity $\theta$ and soil surface $z$ are continuous, then we ask the water velocity $u$ and the water depth $h$ to be also continuous.

Theorem 2.1 (Physical solution). Consider that the parameters $\theta,\lfloor|z|\rfloor$ and $\mathrm{Fr}_{L}$ are fixed. Assume also that equation (46) has the solutions $h_{1}$ and $h_{2}$ such that $h_{1}<\widetilde{h}_{2}<h_{2}$, with $\widetilde{h}_{2}$ given by (48).

Then, the physically relevant solution of the jump equation (46) is given by

$$
h_{0}= \begin{cases}h_{2}, & \text { if } \operatorname{Fr}_{L}<1 \\ h_{1}, & \text { if } \quad \operatorname{Fr}_{L}>1 .\end{cases}
$$

Bounds of the solution. The solution (55) of the jump equation is bounded by

$$
h_{0} \in\left\{\begin{array}{lll}
\left(\widetilde{h}_{2}, h_{m}\right), & \text { if } & \operatorname{Fr}_{L}<1, \\
\left(0, \widetilde{h}_{2}\right), & \text { if } & \operatorname{Fr}_{L}>1,
\end{array}\right.
$$

where

$$
h_{m}=\frac{[a-b(1-\lfloor|z|\rfloor)]+\sqrt{[a-b(1-\lfloor|z|\rfloor)]^{2}-4 b\left[\operatorname{Fr}_{L}^{2}-a(1-\lfloor|z|\rfloor)\right]}}{-2 b} .
$$

Proof. By virtue of the criterion of continuity, the physical relevant solution $h_{0}$ of equation (46) must satisfy the property

$$
h_{0} \longrightarrow 1=\bar{h}_{1} \text {, as } \theta \longrightarrow 1 \text { and }\lfloor|z|\rfloor \longrightarrow 0 .
$$

Using now (52) one can easily obtain $h_{0}$ of the form (55). 
For $\operatorname{Fr}_{L}>1$, we obviously have $h_{0}=h_{1} \in\left(0, \widetilde{h}_{2}\right)$. For $\operatorname{Fr}_{L}<1$, we write

$$
\psi\left(h ; \theta,\lfloor|z|\rfloor, \operatorname{Fr}_{L}\right)=\varphi\left(h ; \theta,\lfloor|z|\rfloor, \operatorname{Fr}_{L}\right) h+\frac{\operatorname{Fr}_{L}^{2}}{\theta}
$$

where

$\varphi\left(h ; \theta,\lfloor|z|\rfloor, \operatorname{Fr}_{L}\right):=-b(\theta) h^{2}-[a(\theta)-b(\theta)(1-\lfloor|z|\rfloor)] h+(1-\lfloor|z|\rfloor) a(\theta)-\operatorname{Fr}_{L}^{2}$.

Since $\varphi\left(h_{m} ; \theta,\lfloor|z|\rfloor, \operatorname{Fr}_{L}\right)=0$ with $h_{m}$ as in $(57)$ and $\psi\left(h_{m} ; \theta,\lfloor|z|\rfloor, \operatorname{Fr}_{L}\right)=$ $\operatorname{Fr}_{L}^{2} / \theta>0$, it follows that $h_{0}=h_{2}<h_{m}$.

\section{Solutions of the Riemann Problem}

From (25), Riemann Problem for the shallow water equations with porosity and topography can be reduced to the problem of finding a solution in the class of bounded variation functions for the following system of equations

$$
\begin{aligned}
\partial_{t}(\theta h)+\partial_{x}(\theta h u) & =0, \\
\partial_{t}(\theta h u)+\partial_{x}\left(\theta h u^{2}\right)+g \theta h \partial_{x}(h+z) & =0,
\end{aligned}
$$

that satisfies the initial datum

$$
(h, u)_{t=0}= \begin{cases}\left(h^{L}, u^{L}\right), & x<0, \\ \left(h^{R}, u^{R}\right), & x>0,\end{cases}
$$

where the terrain data (the porosity $\theta$ and the soil surface $z$ ) are given by

$$
(\theta, z)= \begin{cases}\left(\theta^{L}, z^{L}\right), & x<0 \\ \left(\theta^{R}, z^{R}\right), & x>0\end{cases}
$$

\subsection{Wave solutions}

We build a solution of the problem using rarefaction and shock waves. The rarefaction waves are smooth solutions of the equations (58) in which the porosity $\theta$ is a constant function and the soil surface is a flat horizontal plane $\partial_{x} z=0$. As we discuss in the previously sections, the shock waves solutions verify the generalized Rankine-Hugoniot relations and they can be grouped into two classes:

(a) the shock waves with continuous terrain data, hydrodynamic shock waves (HSW)

and

(b) the shock waves with discontinuous terrain data, terrain shock waves (TSW). 
Although the extended shallow water equations are not hyperbolic equations in the entire domain, we show that at least for a local formulation of the Riemann Problem, one can follow the general framework introduced by Lax [9] for strict hyperbolic system. We introduce three families of solutions that establish the "bricks" of the building block solution of the Riemann Problem. The first two families of solution curves join together the rarefaction waves solutions and HSW. The third family of the solution curves results from the solution of the equation (46) given by (55).

The eigenvalues of the extend system of (58) to the all variables $(h, u, \theta, z)$ are given by

$$
\lambda_{1}=u-\sqrt{g h}, \quad \lambda_{2}=u+\sqrt{g h}, \quad \lambda_{3}=\lambda_{4}=0
$$

and the corresponding eigenvectors are given by

$$
\boldsymbol{r}_{1}=\left(\begin{array}{c}
-\frac{2}{3} \sqrt{\frac{h}{g}} \\
\frac{2}{3} \\
0 \\
0
\end{array}\right), \boldsymbol{r}_{2}=\left(\begin{array}{c}
\frac{2}{3} \sqrt{\frac{h}{g}} \\
\frac{2}{3} \\
0 \\
0
\end{array}\right), \boldsymbol{r}_{3}=\left(\begin{array}{c}
-u^{2} h \\
u g h \\
\theta \mathfrak{f} \\
0
\end{array}\right), \boldsymbol{r}_{4}=\left(\begin{array}{c}
-g h \\
u g \\
0 \\
-\mathfrak{f}
\end{array}\right) .
$$

where $\mathfrak{f}=u^{2}-g h$. The first two eigenvalues $\lambda_{1}$ and $\lambda_{2}$ are genuine nonlinear since $\nabla \lambda_{i} \cdot \boldsymbol{r}_{i}=1, i=1,2$ inside the entire phase space domain $h>0$. The eigenvalues $\lambda_{3}$ and $\lambda_{4}$ are linear degenerated, i.e. $\nabla \lambda_{i} \cdot \boldsymbol{r}_{i}=0, i=3,4$. As one can see, $u^{2}=g h$ are the degeneracy curves for the extended system in the sense that on these curves, the system is not hyperbolic, the eigenvectors $\boldsymbol{r}_{3}$, $\boldsymbol{r}_{4}$ and one of $\boldsymbol{r}_{1}$ or $\boldsymbol{r}_{2}$ collapsing into a single vector.

Denote by $U:=(h, u)$ the hydrodynamic variable and by $W:=(h, u, \theta, z)$ the extended variable. The first two HSW curves are defined as follows.

An $i$-shock wave curve corresponding to the state $W_{0}=\left(h_{0}, u_{0}, \theta_{0}, z_{0}\right)$ is defined by

$$
\mathcal{S}_{i}\left(\epsilon_{i}, W_{0}\right):=\left(\begin{array}{c}
h \\
u \\
\theta \\
z
\end{array}\right)=\left(\begin{array}{l}
h_{0}\left(1+(-1)^{i} \epsilon_{i}\right) \\
u_{0}+\sqrt{g h_{0}} \epsilon_{i} \sqrt{\frac{1}{2}\left(\frac{1}{1+(-1)^{i} \epsilon_{i}}+1\right)} \\
\theta_{0} \\
z_{0}
\end{array}\right), \epsilon_{i} \in(-1,0] .
$$


The shock wave speed $\sigma_{i}$ for the state $U_{0}=\left(h_{0}, u_{0}\right)$ is given by

$\sigma_{i}\left(\epsilon_{i}, U_{0}\right)=u_{0}+(-1)^{i} \sqrt{g h_{0}}\left(1+(-1)^{i} \epsilon_{i}\right) \sqrt{\frac{1}{2}\left(\frac{1}{1+(-1)^{i} \epsilon_{i}}+1\right)}, \epsilon_{i} \in(-1,0]$

and it satisfies

$$
\lambda_{i}(U)<\sigma_{i}<\lambda_{i}\left(U_{0}\right) .
$$

A shock wave solution in the $i$-family is defined by a state $W_{0}$ and a value $\epsilon_{i} \in(-1,0]$ as

$$
(h, u, \theta, z)^{\mathrm{T}}(t, x)= \begin{cases}\left(h_{0}, u_{0}, \theta_{0}, z_{0}\right)^{\mathrm{T}}, & \frac{x}{t}<\sigma_{i}\left(\epsilon_{i}, U_{0}\right) \\ \left(h_{0}\left(1+(-1)^{i} \epsilon_{i}\right), u\left(\epsilon_{i}\right), \theta_{0}, z_{0}\right), & \frac{x}{t}>\sigma_{i}\left(\epsilon_{i}, U_{0}\right)\end{cases}
$$

where $u\left(\epsilon_{i}\right)$ and $\sigma_{i}\left(\epsilon_{i}, U_{0}\right)$ are given by (63) and (64), respectively.

An $i$-rarefaction wave curve is defined by

$$
\mathcal{R}_{i}\left(\epsilon_{i}, W_{0}\right):=\left(\begin{array}{l}
h \\
u \\
\theta \\
z
\end{array}\right)=\left(\begin{array}{l}
h_{0}\left(1+(-1)^{i} \epsilon_{i}\right) \\
u_{0}+(-1)^{i} 2 \sqrt{g h_{0}}\left(\sqrt{1+(-1)^{i} \epsilon_{i}}-1\right) \\
\theta_{0} \\
z_{0}
\end{array}\right), \epsilon_{i} \geq 0 .
$$

The velocity component of a rarefaction $\mathcal{R}_{i}\left(\epsilon_{i}, W_{0}\right)$ wave solution is defined by

$$
u(t, x)= \begin{cases}u_{0}, & \frac{x}{t}<\lambda_{i}\left(U_{0}\right) \\ u_{0}+\frac{2}{3}\left(\frac{x}{t}-\lambda_{i}\left(U_{0}\right)\right), & \lambda_{i}\left(U_{0}\right)<\frac{x}{t}<\lambda_{i}\left(U\left(\epsilon_{i}\right)\right) \\ u\left(\epsilon_{i}\right), & \frac{x}{t}>\lambda_{i}\left(U\left(\epsilon_{i}\right)\right)\end{cases}
$$

where $u\left(\epsilon_{i}\right)$ is given by $(67)$. The $h$ component of the rarefaction wave solution is

$$
h(t, x)=\frac{1}{g}\left(\sqrt{g h_{0}}+(-1)^{i}\left(u(t, x)-u_{0}\right) / 2\right)^{2}
$$

and the terrain components are $(\theta, z)^{\mathrm{T}}=\left(\theta_{0}, z_{0}\right)^{\mathrm{T}}$.

One defines an $i$-wave as

$$
\mathcal{W}_{i}\left(\epsilon_{i}, W_{0}\right)=\left\{\begin{array}{l}
\mathcal{S}_{i}\left(\epsilon_{i}, W_{0}\right), \epsilon_{i}<0 \\
\mathcal{R}_{i}\left(\epsilon_{i}, W_{0}\right), \epsilon_{i} \geq 0
\end{array}\right.
$$


Note that an $i$-wave curve is of class $C^{1}$ with respect to its argument and

$$
\left.\frac{\mathrm{d}}{\mathrm{d} \epsilon_{i}} \mathcal{W}_{i}\left(\epsilon_{i}, W_{0}\right)\right|_{\epsilon_{i}=0}=\frac{3}{2} \sqrt{g h_{0}} \boldsymbol{r}_{i}\left(U_{0}\right)
$$

We emphasize that terrain components of a solution induced by any member of $\mathcal{W}_{i}, i=1,2$ are constant functions.

In order to build a solution that takes into account the jump of the soil surface or the porosity, we introduce a third family of shock waves generated by the solutions of the generalized Rankine-Hugoniot relations (31).

Rewrite (41) as

$$
-b\left(\epsilon_{3}\right) h^{3}-\left(a\left(\epsilon_{3}\right)-b\left(\epsilon_{3}\right)\left(1-\epsilon_{4}\right)\right) h^{2}+\left(\left(1-\epsilon_{4}\right) a\left(\epsilon_{3}\right)-\mathrm{Fr}_{0}^{2}\right) h+\frac{\operatorname{Fr}_{0}^{2}}{\epsilon_{3}}=0
$$

where $\operatorname{Fr}_{0}^{2}=\frac{u_{0}^{2}}{g h_{0}}$ and the functions $a$ and $b$ are extended by continuity at $\epsilon_{3}=1$.

From proposition 2.2 we know that if $\operatorname{Fr}_{0} \neq 1$, then there exists a neighborhood of 1 for $\epsilon_{3}$ and a neighborhood of 0 for $\epsilon_{4}$ such that the equation has a solution in the neighborhood of 1 . We denote by $\beta\left(\epsilon_{3}, \epsilon_{4}\right)$ the solution defined the theorem 2.1. Thus, there exists a function $\beta\left(\epsilon_{3}, \epsilon_{4}\right)$ solution of the equation (70) which in addition satisfies

$$
\lim _{\epsilon_{3} \rightarrow 1, \epsilon_{4} \rightarrow 0} \beta\left(\epsilon_{3}, \epsilon_{4}\right)=1 .
$$

By simple standard computations, one can show that

$$
\left.\frac{\partial \beta\left(\epsilon_{3}, \epsilon_{4}\right)}{\partial \epsilon_{3}}\right|_{\epsilon_{3}=1, \epsilon_{4}=0}=\frac{\operatorname{Fr}_{0}^{2}}{1-\mathrm{Fr}_{0}^{2}},\left.\quad \frac{\partial \beta\left(\epsilon_{3}, \epsilon_{4}\right)}{\partial \epsilon_{4}}\right|_{\epsilon_{3}=1, \epsilon_{4}=0}=\frac{1}{\operatorname{Fr}_{0}^{2}-1}
$$

Then, using (38) and (40), we define the terrain shock waves family $\mathcal{W}_{3}\left(\epsilon_{3}, \epsilon_{4}, W_{0}\right)$ as

$$
\mathcal{W}_{3}\left(\epsilon_{3}, \epsilon_{4}, W_{0}\right):=\left(\begin{array}{c}
h \\
u \\
\theta \\
z
\end{array}\right)=\left(\begin{array}{l}
\beta\left(\epsilon_{3}, \epsilon_{4}\right) h_{0} \\
\frac{u_{0}}{\epsilon_{3} \beta\left(\epsilon_{3}, \epsilon_{4}\right)} \\
\epsilon_{3} \theta_{0} \\
z_{0}+\epsilon_{4} h_{0}
\end{array}\right), \epsilon_{3} \in \mathcal{V}_{1}(1), \epsilon_{4} \in \mathcal{V}_{2}(0) .
$$

Note also that the gradient of $\mathcal{W}_{3}$ is given by

$$
\begin{aligned}
& \left.\partial_{\epsilon_{3}} \mathcal{W}_{3}\left(\epsilon_{3}, \epsilon_{4}, W_{0}\right)\right|_{\epsilon_{3}=1, \epsilon_{4}=0}=\frac{1}{g h_{0}\left(\mathrm{Fr}_{0}^{2}-1\right)} \boldsymbol{r}_{3}\left(W_{0}\right), \\
& \left.\partial_{\epsilon_{4}} \mathcal{W}_{3}\left(\epsilon_{3}, \epsilon_{4}, W_{0}\right)\right|_{\epsilon_{3}=1, \epsilon_{4}=0}=\frac{-h_{0}}{g h_{0}\left(\mathrm{Fr}_{0}^{2}-1\right)} \boldsymbol{r}_{4}\left(W_{0}\right) .
\end{aligned}
$$


A terrain shock wave solution is defined by

$$
\left(\begin{array}{l}
h \\
u \\
\theta \\
z
\end{array}\right)(t, x)= \begin{cases}W_{0}, & x<0, t>0 \\
\mathcal{W}_{3}\left(\epsilon_{3}, \epsilon_{4}, W_{0}\right), & x>0, t>0\end{cases}
$$

Remark that there exists an essential difference between a steady HSW $i$-shock wave and a terrain shock wave. Although, $x=0$ is a discontinuity line for both shock waves, only the second one bears jump in terrain data.

Using these three families of waves, we can now analyze the solution of the Riemann Problem. In the next subsection, we discuss the local Riemann Problem and then we analyze the global problem.

\subsection{Local solutions}

The jump in the terrain data will induce a discontinuous line $x=0$ in the solution of the shallow water equations. This fact precludes a rarefaction wave to cross the discontinuity line. It must be located entirely on left or on right of the line $x=0$. On the other hand, a shock wave emerging from $x=0$ propagates either on left or on right of $x=0$. Keeping in mind that we follow a classical way to build up a solution of Riemann Problem $[9,4,11]$, let $W_{L}$ be given the left state of the Riemann Problem and $\mathcal{V}_{a}, a=1,4$ certain neighborhoods introduced as in the definition of the wave curve $\mathcal{W}_{i}, i=1,3$. Let $\Omega\left(W_{0}\right)$ be a neighborhood of $W_{0}$. One defines the application

$$
\mathcal{W}_{i_{1}} \circ \mathcal{W}_{i_{2}} \circ \mathcal{W}_{i_{3}}\left(\cdot, \cdot, \cdot, W_{0}\right): \underset{a=1,4}{\times} \mathcal{V}_{a} \rightarrow \Omega\left(W_{0}\right)
$$

where the composition operator $\circ$ is defined as

$$
\mathcal{W}_{i_{1}} \circ \mathcal{W}_{i_{2}}\left(\epsilon_{i_{1}}, \epsilon_{i_{2}}, W_{0}\right)=\mathcal{W}_{i_{1}}\left(\epsilon_{i_{1}}, \mathcal{W}_{i_{2}}\left(\epsilon_{i_{2}}, W_{0}\right)\right)
$$

The order in the composition of the elementary waves $\mathcal{W}_{i}$ is governed by the increasing order of the eigenvalues of the system of the equations. In the case of strict hyperbolicity problem, there is no problem to define the composition operation. But in the case of resonant problem, when one or more eigenvalues become equal, the increasing order can change.

Depending on the position of $W_{0}$ in the phase space, one encounters the following situations

$$
\begin{array}{ll}
\text { (I) } & \mathcal{W}_{2} \circ \mathcal{W}_{1} \circ \mathcal{W}_{3}, \\
(\mathrm{II}) & \mathcal{W}_{2} \circ \mathcal{W}_{3} \circ \mathcal{W}_{1}, \\
(\mathrm{III}) & \mathcal{W}_{3} \circ \mathcal{W}_{2} \circ \mathcal{W}_{1} .
\end{array}
$$


We show that if $U^{L}$ is such that the Froude number $\operatorname{Fr}_{L}>1$ or $\operatorname{Fr}_{L}<1$, then there exists a neighborhood $\Omega\left(W^{L}\right)$ such that for any $W^{R} \in \Omega\left(W^{L}\right)$ the Riemann Problem is solvable.

Lemma 3.1. Let $m^{2}<1$ be a given real number. Then, there exists $\rho_{1}$ and $\rho_{2}$ real and positives numbers such that for any $\theta \in\left(1-\rho_{1}, 1+\rho_{1}\right)$ and $\lfloor|z|\rfloor \in\left(-\rho_{2}, \rho_{2}\right)$ and $\operatorname{Fr}^{2}<m^{2}$ the following properties hold:

1. The equation

$$
\psi(h ; \theta,\lfloor|z|\rfloor, \operatorname{Fr})=0
$$

admits two positive solutions.

2. The Froude number associated to the right state satisfy the inequality

$$
\operatorname{Fr}_{R}\left(\theta, z, F_{L}\right)<1 \text {. }
$$

Proof. 1. The existence of the solution of jump equation for any $\mathrm{Fr}^{2} \neq 1$ was proved by the Proposition 2.2. Here we prove that there exist certain uniform bounds for $\theta$ and $\lfloor|z|\rfloor$ with respect to Froude number. To show the existence of the solution, we essentially use the continuity of the roots of a polynomial function w.r.t. the coefficients of the polynomial. The coefficients $a(\theta)$ and $b(\theta)$ are continuous functions with respect to $\theta$.

First, consider $\mathrm{Fr}=m$. For this fixed value of the Froude number, by Proposition 2.2 (c) we know that we can find two numbers $\rho_{1}, \rho_{2}>0$ such that equation (78) with $\mathrm{Fr}=m$ has 2 positive solutions, for any $\theta \in\left(1-\rho_{1}, 1+\rho 1\right)$ and $\lfloor|z|\rfloor \in\left(-\rho_{2}, \rho_{2}\right)$.

In order to show the first statement of this Lemma, we split the proof into two steps with complementary assumptions:

CASE 1. $\frac{1}{\theta}<1-\lfloor|z|\rfloor$.

In this case, using Proposition 2.2 (b) we find that equation (78) has solution for any

$$
(\theta,\lfloor|z|\rfloor) \in\left(1-\rho_{1}, 1+\rho_{1}\right) \times\left(-\rho_{2}, \rho_{2}\right) \cap\{(\theta,\lfloor|z|\rfloor) \mid 1 / \theta<1-\lfloor|z|\rfloor\} .
$$

CASE 2. $\frac{1}{\theta}>1-\lfloor|z|\rfloor$.

For $\operatorname{Fr}=m$, consider the wave solution $\beta(m):=\beta(\theta,\lfloor|z|\rfloor, m)$. We can see that

$$
\begin{aligned}
\psi(\beta(m), \theta,[|z|], \operatorname{Fr}) & =\psi(\beta(m), \theta,[|z|], \operatorname{Fr})-\psi(\beta(m), \theta,[|z|], m)= \\
& =\left(\operatorname{Fr}^{2}-m^{2}\right)\left(\frac{1}{\theta}-\beta(m)\right) .
\end{aligned}
$$


To prove the existence of the solutions in this case, it is sufficient to show that $1 / \theta>\beta(m)$. For this purpose, we compute the derivative of $\partial_{h} \psi(1 / \theta, \theta,\lfloor|z|\rfloor, m)$ and write it in the following form

$$
\partial_{h} \psi(1 / \theta, \theta,\lfloor|z|\rfloor, m)=-m^{2}+g(\theta,\lfloor|z|\rfloor),
$$

where

$$
g(\theta,\lfloor|z|\rfloor)=\frac{1}{\theta}+\left(\frac{1}{\theta}-1+\lfloor|z|\rfloor\right)\left(-\frac{b(\theta)}{\theta}+1\right) .
$$

Let $\alpha$ be some positive number such that $m^{2}<1-\alpha$. Since the function $g$ is continuous and $g(1,0)=1$, we can restrict the values of $\rho_{1}$ and $\rho_{2}$ such that $g(\theta,\lfloor|z|\rfloor)>1-\alpha / 2$ for any $\theta \in\left(1-\rho_{1}, 1+\rho_{1}\right)$ and $\lfloor|z|\rfloor \in\left(-\rho_{2}, \rho_{2}\right)$. Consequently, $\partial_{h} \psi(1 / \theta, \theta,\lfloor|z|\rfloor, m)>\alpha / 2>0$, for any $\theta \in\left(1-\rho_{1}, 1+\rho_{1}\right)$ and $\lfloor|z|\rfloor \in\left(-\rho_{2}, \rho_{2}\right)$. On the other hand, for $1 / \theta>1-\lfloor|z|\rfloor$ we can immediately verify that

$$
\psi(1 / \theta, \theta,[|z|], m)>0 .
$$

and consequently, $1 / \theta>\beta(m)$. Then, equation (78) has solution for any

$$
(\theta,\lfloor|z|\rfloor) \in\left(1-\rho_{1}, 1+\rho_{1}\right) \times\left(-\rho_{2}, \rho_{2}\right) \cap\{(\theta,\lfloor|z|\rfloor) \mid 1 / \theta>1-\lfloor|z|\rfloor\} .
$$

CASE 3. $\frac{1}{\theta}=1-\lfloor|z|\rfloor$.

In this case, one can easily verify that equation (78) has always two positive solutions

$$
h_{1}=\frac{-a(\theta)-\sqrt{a(\theta)^{2}-4 b(\theta) \mathrm{Fr}^{2}}}{2 b(\theta)}, \quad h_{2}=\frac{1}{\theta} .
$$

2. We prove inequality (79) by analyzing the three cases previously discussed.

CASE 1. $\frac{1}{\theta}<1-\lfloor|z|\rfloor$.

One has

$$
\begin{aligned}
\psi(h ; \theta,\lfloor|z|\rfloor, \mathrm{Fr}):= & -b(\theta) h^{3}-[a(\theta)-b(\theta)(1-\lfloor|z|\rfloor)] h^{2}+ \\
& +\left[(1-\lfloor|z|\rfloor) a(\theta)-\mathrm{Fr}^{2}\right] h+\frac{\mathrm{Fr}^{2}}{\theta} .
\end{aligned}
$$

and since $a(\theta), b(\theta)$ are negative, then $\psi(1 / \theta ; \theta,\lfloor|z|\rfloor, \operatorname{Fr})<0$. In this case, the solutions are separated by $1 / \theta$. Consequently,

$$
\beta\left(\theta,\lfloor|z|\rfloor, \operatorname{Fr}_{L}\right)>\frac{1}{\theta} .
$$

CASE 2. $\frac{1}{\theta}>1-\lfloor|z|\rfloor$. 
It is easily verified that

$$
\begin{aligned}
\psi(\beta(\theta,[|z|], m), \theta,[|z|], \operatorname{Fr}) & =\psi(\beta(\theta,[|z|], m), \theta,[|z|], \operatorname{Fr})- \\
& -\psi(\beta(\theta,[|z|], m), \theta,[|z|], m)= \\
& =\left(\operatorname{Fr}^{2}-m^{2}\right)\left(\frac{1}{\theta}-\beta(\theta,[|z|], m)\right) .
\end{aligned}
$$

Since $1 / \theta>1-\lfloor|z|\rfloor$ and $\mathrm{Fr}^{2}<m^{2}$, one has $\psi(\beta(\theta,[|z|], m), \theta,[|z|], \operatorname{Fr})<0$. Then, the two positive solutions are separated by $\beta(\theta,[|z|], m)$ and

$$
\beta\left(\theta,\lfloor|z|\rfloor, \operatorname{Fr}_{L}\right)>\beta(\theta,[|z|], m) .
$$

CASE 3. $\frac{1}{\theta}=1-\lfloor|z|\rfloor$.

Using the fact that one positive solution is $1 / \theta$ in this case, one immediately gets

$$
\beta\left(\theta,\lfloor|z|\rfloor, \operatorname{Fr}_{L}\right)>\frac{1}{\theta} .
$$

Let

$$
\gamma(\theta,[|z|], m):=\min \left\{\frac{1}{\theta}, \beta(\theta,[|z|], m)\right\}
$$

and denote by $\gamma(m)$ the infimum of $\gamma(\theta,[|z|], m)$ on the set of elements $(\theta,\lfloor|z|\rfloor) \in\left(1-\rho_{1}, 1+\rho_{1}\right) \times\left(-\rho_{2}, \rho_{2}\right)$.

Using inequalities (84), (86) and (87), we can see that $\gamma(m)$ represents an inferior bound of $\beta\left(\theta,\lfloor|z|\rfloor, \operatorname{Fr}_{L}\right)$. Also (38) and (40) give

$$
\operatorname{Fr}_{R}^{2}\left(\theta,[|z|] \operatorname{Fr}_{L}\right)=\frac{\operatorname{Fr}_{L}^{2}}{\theta^{2} \beta^{3}\left(\theta,[|z|] \operatorname{Fr}_{L}\right)}<\frac{m^{2}}{\left(1-\rho_{1}\right)^{2} \gamma^{3}(m)}
$$

and since

$$
\lim _{\theta \rightarrow 1,\lfloor|z|\rfloor \rightarrow 0} \gamma(\theta,\lfloor|z|\rfloor, m)=1,
$$

one can restrict $\rho_{1}$ and $\rho_{2}$ to values such that the last expression in (88) to be subunitary. This proves relation (79).

In what follows, we will use the following notations

$$
U:=(h, u), \quad U^{L}:=\left(h^{L}, u^{L}\right), \quad U^{R}:=\left(h^{R}, u^{R}\right), \quad \operatorname{Fr}^{2}(U):=\frac{u^{2}}{g h} .
$$

We obviously have $\operatorname{Fr}_{L}^{2}=\operatorname{Fr}^{2}\left(U^{L}\right)$ and $\operatorname{Fr}_{R}^{2}=\operatorname{Fr}^{2}\left(U^{R}\right)$. 
Theorem 3.1. Consider that $\theta^{L}$ and $z^{L}$ are given and let $U^{L}=\left(h^{L}, u^{L}\right)$ be such that $\operatorname{Fr}_{L}^{2} \neq 1$. Then, there exists a neighborhood $\Omega\left(W^{L}\right)$ such that if $W^{R} \in \Omega\left(W^{L}\right)$, then the Riemann Problem

$$
\begin{aligned}
& \frac{\partial}{\partial t} \theta h+\partial_{x} \theta h u=0 \\
& \frac{\partial}{\partial t} \theta h u+\partial_{x} \theta h u^{2}+g \theta h \partial_{x}(z+h)=0 \\
& \frac{\partial}{\partial t} \theta=\frac{\partial}{\partial t} z= 0, \\
&\left.(h, u, \theta, z)^{\mathrm{T}}\right|_{t=0}= \begin{cases}\left(h^{L}, u^{L}, \theta^{L}, z^{L}\right)^{\mathrm{T}}, & x<0 \\
\left(h^{R}, u^{R}, \theta^{R}, z^{R}\right)^{\mathrm{T}}, & x>0\end{cases}
\end{aligned}
$$

has at least one solution.

Proof. Depending on $U^{L}$, we find ourselves in one of the following three distinct situations

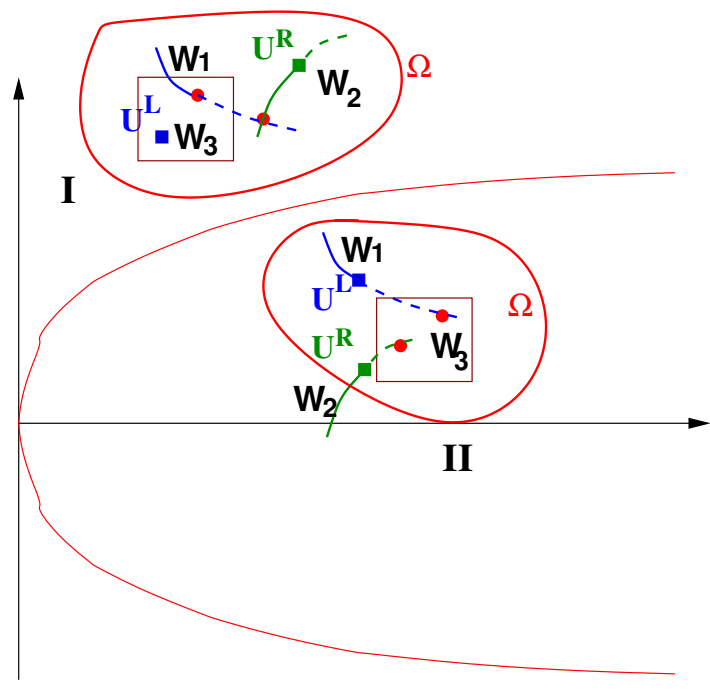

III

Figure 3: The local configuration of Riemann Problem.

(I) $\operatorname{Fr}_{L}>1$ and $u^{L}>0$,

(II) $\operatorname{Fr}_{L}<1$,

(III) $\operatorname{Fr}_{L}>1$ and $u^{L}<0$, 
pictured in Figure 3. We only analyze the case (II) since the other two cases can be similarly resolved.

First, for any $\eta_{1}, \eta_{2}, \eta_{3}$ and $\eta_{4}$ positive numbers, let us define the intervals

$$
\begin{array}{ll}
\mathcal{V}\left(\eta_{1}\right):=\left(-\eta_{1}, \eta_{1}\right), & \mathcal{V}\left(\eta_{2}\right):=\left(-\eta_{2}, \eta_{2}\right), \\
\mathcal{V}\left(\eta_{3}\right):=\left(1-\eta_{3}, 1+\eta_{3}\right), & \mathcal{V}\left(\eta_{4}\right):=\left(-\eta_{4}, \eta_{4}\right) .
\end{array}
$$

We will also use the following notations:

$$
\begin{aligned}
& \varepsilon:=\left(\epsilon_{2}, \epsilon_{3}, \epsilon_{4}, \epsilon_{1}\right), \\
& \varepsilon_{0}:=(0,1,0,0), \\
& \Gamma_{\boldsymbol{\eta}}:=\mathcal{V}\left(\eta_{2}\right) \times \mathcal{V}\left(\eta_{3}\right) \times \mathcal{V}\left(\eta_{4}\right) \times \mathcal{V}\left(\eta_{1}\right) .
\end{aligned}
$$

We first show that there exists a set of positive numbers $\eta_{1}, \eta_{2}, \eta_{3}$ and $\eta_{4}$ such that the application

$$
\mathcal{W}_{I I}\left(\varepsilon ; W^{L}\right):=\mathcal{W}_{2}\left(\epsilon_{2}, \mathcal{W}_{3}\left(\epsilon_{3}, \epsilon_{4}, \mathcal{W}_{1}\left(\epsilon_{1}, W^{L}\right)\right)\right)
$$

defines a weak solution of the extended shallow water equation system for any $\varepsilon \in \Gamma_{\boldsymbol{\eta}}$.

One defines the bounds $\delta_{1}$ and $\delta_{2}(U)$

$$
\delta_{1}=1-\left(\frac{\operatorname{Fr}_{L}+2}{3}\right)^{2}, \quad \delta_{2}(U)=\frac{-3+\sqrt{1+8 \operatorname{Fr}_{U}^{2}}}{2},
$$

such that if $\epsilon_{1}<\delta_{1}$, then $\sigma_{1}\left(\epsilon_{1}, U^{L}\right)<0$ or $\lambda_{1}\left(\epsilon_{1}, U^{L}\right)<0$ (the solution induced by $\mathcal{W}_{1}\left(\epsilon_{1}, U^{L}\right)$ is on the left side of $\left.x=0\right)$. If $\epsilon_{2}>\delta_{2}(U)$ and $\operatorname{Fr}(U)<$ 1 , then $\lambda_{2}(U)>0$ and $\sigma_{2}\left(\epsilon_{2}, U\right)>0$ (the solution induced by $\mathcal{W}_{2}\left(\epsilon_{2}, U\right)$ is on the right side of $x=0)$.

Now let $h\left(\epsilon_{1}\right)$ and $\operatorname{Fr}\left(\epsilon_{1}\right)$ be the quantities evaluated on the state $\mathcal{W}_{1}\left(\epsilon_{1}, W^{L}\right)$. Let also $\eta_{1}>0$ be such that $\eta_{1}<\delta_{1}$ and $\operatorname{Fr}\left(\epsilon_{1}\right)<1$ for any $\epsilon_{1} \in \mathcal{V}_{1}\left(\eta_{1}\right)$.

Using Lemma 3.1, we can find $\eta_{3}>0$ and $\eta_{4}>0$ such that the terrain wave equation has a solution $\beta\left(\epsilon_{3}, \epsilon_{4}\right)$ for any $\epsilon_{3} \in \mathcal{V}\left(\eta_{3}\right)$ and $\epsilon_{4} \in \mathcal{V}\left(\eta_{4}\right)$, solution that in addition satisfies $\operatorname{Fr}\left(\epsilon_{3}, \epsilon_{4}, \epsilon_{1}\right)<1$, where $\operatorname{Fr}\left(\epsilon_{3}, \epsilon_{4}, \epsilon_{1}\right)$ is Froude number associated to the state $\mathcal{W}_{3}\left(\epsilon_{3}, \epsilon_{4} ; \mathcal{W}_{1}\left(\epsilon_{1}, W^{L}\right)\right)$.

In order to define the application $\mathcal{W}_{2}\left(\epsilon_{2}, \mathcal{W}_{3}\left(\epsilon_{3}, \epsilon_{4} ; \mathcal{W}_{1}\left(\epsilon_{1}, W^{L}\right)\right)\right)$, the parameter $\epsilon_{2}$ must satisfy the relation

$$
\epsilon_{2}>\delta_{2}\left(U\left(\epsilon_{3}, \epsilon_{4}, \epsilon_{1}\right)\right)=\frac{-3+\sqrt{1+8 \operatorname{Fr}^{2}\left(\epsilon_{3}, \epsilon_{4}, \epsilon_{1}\right)}}{2} .
$$

From continuity of the right hand side of the above inequality, one can find $\eta_{2}$ and $\eta_{1}, \eta_{3}, \eta_{4}$ such that

$$
-\eta_{2}>\frac{-3+\sqrt{1+8 \operatorname{Fr}^{2}\left(\epsilon_{3}, \epsilon_{4}, \epsilon_{1}\right)}}{2}
$$


for any $\epsilon_{1} \in \mathcal{V}\left(\eta_{1}\right), \epsilon_{3} \in \mathcal{V}\left(\eta_{3}\right), \epsilon_{4} \in \mathcal{V}\left(\eta_{4}\right)$.

Now, using the found bounds $\eta_{i}, i=\overline{1,4}$, one can define the application

$$
\mathcal{W}_{I I}\left(\varepsilon ; W^{L}\right): \Gamma_{\eta} \rightarrow \Omega\left(W^{L}\right) .
$$

It is clear that

$$
\mathcal{W}_{I I}\left(\varepsilon_{0} ; W^{L}\right)=W^{L}
$$

and that the gradient of $\mathcal{W}_{I I}\left(\varepsilon ; W^{L}\right)$ with respect to $\varepsilon$ is

$$
\left.\partial_{\varepsilon} \mathcal{W}_{I I}\left(\varepsilon ; W^{L}\right)\right|_{\varepsilon=0}=\left[\mathfrak{g} \boldsymbol{r}_{2}\left(W^{L}\right), \mathfrak{f} \boldsymbol{r}_{3}\left(W^{L}\right),-h^{L} \mathfrak{f} \boldsymbol{r}_{4}\left(W^{L}\right), \mathfrak{g} \boldsymbol{r}_{1}\left(W^{L}\right)\right]
$$

where

$$
\mathfrak{g}=3 / 2 \sqrt{g h^{L}} \quad \text { and } \quad \mathfrak{f}=\frac{1}{g h\left(\mathrm{Fr}_{L}^{2}-1\right)} .
$$

Since the eigenvectors $\boldsymbol{r}_{i}, i=\overline{1,4}$ are linear independent for $\operatorname{Fr}_{L}^{2} \neq 1$, one can now invoke the implicit function theorem and shrink the neighborhood $\Gamma_{\boldsymbol{\eta}}$ such that $\mathcal{W}_{I I}$ is a one-to-one application from $\Gamma_{\boldsymbol{\eta}}$ to $\Omega\left(W^{L}\right)$.

Figure 4 exemplifies the existence of the solution of the Riemann Problem for $\operatorname{Fr}_{L}<1$. From this picture in the phase space $(h, u)$, one can observe

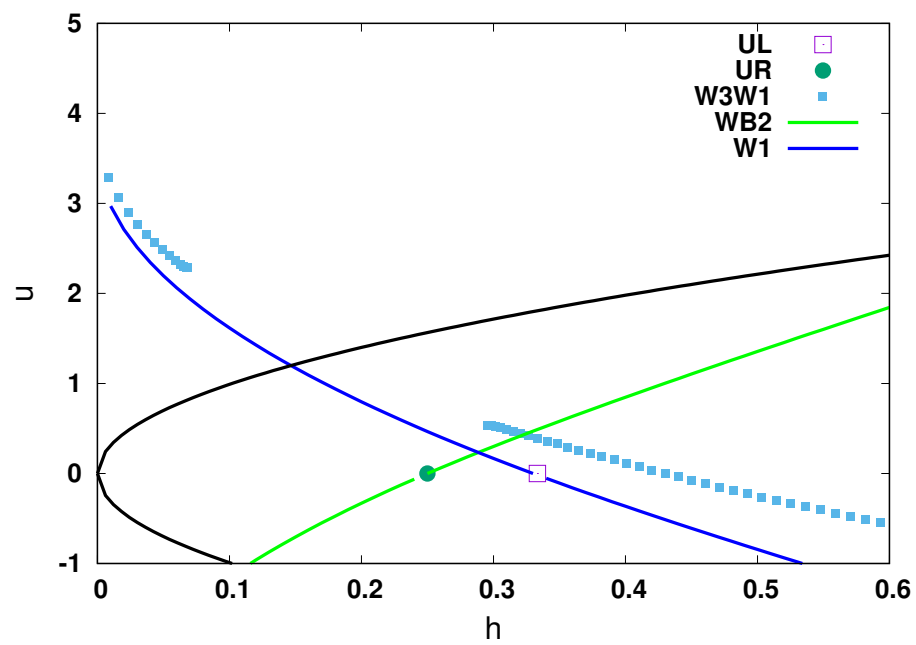

Figure 4: The solution in the phase space of a Riemann Problem.

that in order for the solution to exist, it is essential that the $\mathcal{W}_{2}$ type wave to 
intersect the composition of $\mathcal{W}_{3}$ with $\mathcal{W}_{1}$. The initial states $L$ and $R$ for this Riemann Problem are given

$$
\begin{array}{lll}
h^{L}=0.33 & \theta^{L}=0.9 & z^{L}=1.0 \\
h^{R}=0.25 & \theta^{L}=1.0 & z^{L}=0.9
\end{array} \quad u^{L}=u^{R}=0
$$

Note that the terrain characteristics $\epsilon_{3}$ and $\epsilon_{4}$ are fixed. In order to build the solution of the Riemann Problem, one must "walk" from $L$ to $R$ by varying $\epsilon_{1}$ and $\epsilon_{2}$. This "walk" is achieved as follows. We start from $W^{L}$ and we walk on $\mathcal{W}_{1}$ by varying $\epsilon_{1}$ (if $\epsilon_{1}>0$ then we move up on the simple wave $\mathcal{W}_{1}$; if $\epsilon_{1}<0$ then we move down on the shock wave $\mathcal{W}_{1}$ ). During this time of varying $\epsilon_{1}$, the curve $\mathcal{W}_{3}\left(\epsilon_{3}, \epsilon_{4}, \mathcal{W}_{1}\left(\epsilon_{1}, W^{L}\right)\right)$ is formed since $\epsilon_{3}$ and $\epsilon_{4}$ are fixed. On this curve $\mathcal{W}_{3}$ one must find the point that corresponds to the intersection with the backward curve $\mathcal{W}_{2}\left(\epsilon_{2}, \mathcal{W}_{3}\right)$. Once this accomplished, then one can build the solution $\mathcal{W}_{2}\left(\epsilon_{2}, \mathcal{W}_{3}\left(\epsilon_{3}, \epsilon_{4}, \mathcal{W}_{1}\left(\epsilon_{1}, W^{L}\right)\right)\right)$ for which the free surface is drawn in Figure 5. Note the solution after an amount of time $t=0.25$ built (from left to right) with the simple (rarefaction) wave $\mathcal{W}_{1}$, the terrain wave $\mathcal{W}_{3}$ and the shock wave $\mathcal{W}_{2}$

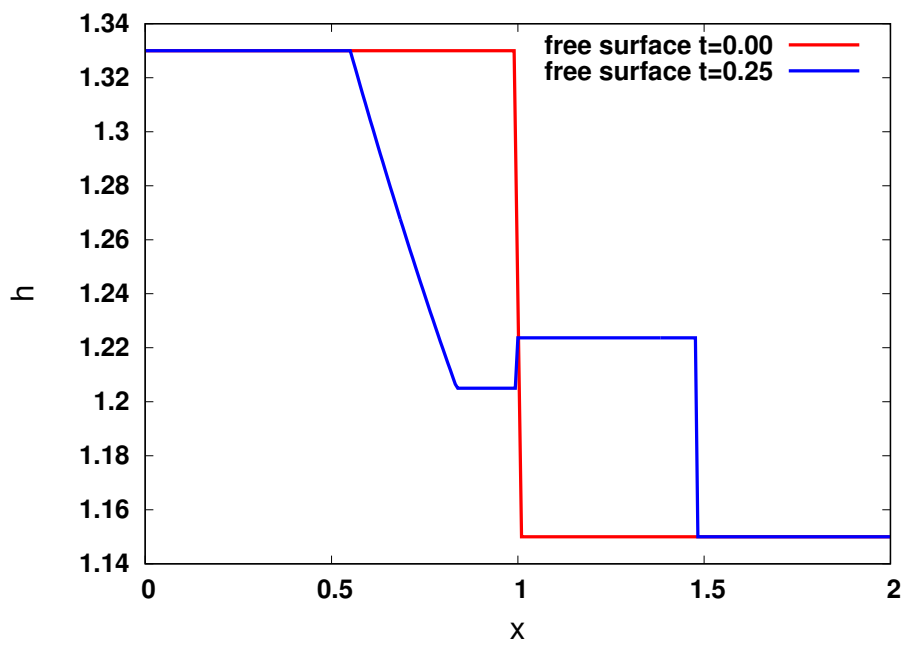

Figure 5: The free surface associated to the solution from 4.

\section{Conclusion and Remarks}

In this paper, we solved the Riemann Problem for shallow water with vegetation. 
- The path connecting curve necessary to define the shock wave solution of a nonconservative hyperbolic system was build based on physical arguments.

- We proved the existence of the solution of the Riemann Problem for hyperbolic systems which are not strictly hyperbolic.

- We believe that the following conjecture holds: the local existence theorem holds regardless of the path.

\section{Acknowledgment}

This work was performed within the project 50/2012 ASPABIR (www.aspabir.biogeochemistry.ro) funded by UEFISCDI, Romania.

\section{References}

[1] L. Ambrosio, N. Fusco, D. Pallara, Functions of Bounded Variation and Free Discontinuity Problems," Oxford Mathematical Monographs, The Clarendon Press, Oxford University Press, New York, 2000.

[2] S. Bianchini, A. Bressan, Vanishing viscosity solutions of nonlinear hyperbolic systems, Ann. of Math., 161(2005), 223-342.

[3] F. Bouchut, Nonlinear Stability of Finite Volume Methods for Hyperbolic Conservation Laws and Well-Balanced Schemes for Sources, Frontiers in Mathematics, Birkhauser Verlag, Basel, 2004.

[4] C. M. Dafermos, Solution of the Riemann problem for a class of hyperbolic systems of conservation laws by the viscosity method, Arch. Rational Mech. Anal., 52(1973), 1-9.

[5] G. Dal Maso, P.G. LeFloch, F. Murat, Definition and weak stability of nonconservative products, J. Math. Pures Appl., 74(1995), 483-548.

[6] R. DiPerna, Measure-valued solutions of conservation laws, Arch. Rat. Mech. Anal. 88(1985), 223-270.

[7] Thierry Gallouet, Jean-Marc Herard, Nicolas Seguin, Some approximate Godunov schemes to compute shallow-water equations with topography, Computers\&Fluids, 32(2003) 479-513.

[8] J. Glimm, Solutions in the large for nonlinear hyperbolic systems of equations, Commun. Pure. Appl. Math. 18(1965), 697-715.

[9] P.D. Lax, Hyperbolic systems of conservation laws. II., Commun. Pure Appl. Math. 10(1957), 537-566. 
[10] P.G. LeFloch, Mai Duc Thanh, Rieamann problem for the shallow water equations with discountinous topography, Commun. Pure Appl. Math. $\mathbf{5}(4)(2007), 865-885$.

[11] P.G. LeFloch, Hyperbolic systems of conservation laws: the theory of classical and nonclassical shock waves, Lectures in Mathematics, ETH Zuerich, Birkhauser, 2002.

[12] J.A. Smoller, Shock Waves and Reaction-Diffusion Equations, (Second Edition). New York: Springer, 1994.

[13] E.F. Toro, Riemann solvers and numerical methods for fluid dynamics, Berlin: Springer, 1997.

[14] A.I. Volpert, The spaces $B V$ and quasilinear equations, Math. USSR Sbornik, 73(1967), 255-302.

Stelian ION, Email: stelian.ion@ima.ro

Dorin MARINESCU, Email: dorin.marinescu@ima.ro

Stefan-Gicu CRUCEANU, Email: stefan.cruceanu@ima.ro

Institute of Statistical Mathematics and Applied Mathematics

Romanian Academy

Calea 13 Septembrie, No. 13, 050711 Bucharest, Romania. 